\title{
Higher-Order Quasicontinuum Methods for Elastic and Dissipative Lattice Models: Uniaxial Deformation and Pure Bending
}

\author{
L.A.A. Beex ${ }^{1, *}$, O. Rokoš ${ }^{2}$, J. Zeman ${ }^{2}$, and S.P.A. Bordas ${ }^{1,3}$ \\ ${ }^{1}$ University of Luxembourg, Research Unit of Engineering Science, Campus Kirchberg \\ 6 rue Richard Coudenhove-Kalergi, L-1359 Luxembourg, Luxembourg. \\ ${ }^{2}$ Czech Technical University in Prague, Faculty of Civil Engineering, Thákurova 7, 16629 \\ Prague 6, Czech Republic. \\ ${ }^{3}$ Cardiff University, School of Engineering, Queen's Buildings, The Parade, Cardiff CF24 \\ 3AA, Wales, UK.
}

Received XXXX, revised XXXX, accepted XXXX

Published online XXXX

Key words Quasicontinuum method, Multiscale, Lattice model, Higher-order, Pure bending.

The quasicontinuum (QC) method is a numerical strategy to reduce the computational cost of direct lattice computations - in this study we achieve a speed up of a factor of 40 . It has successfully been applied to (conservative) atomistic lattices in the past, but using a virtualpower statement it was recently shown that QC approaches can also be used for spring and beam lattice models that include dissipation. Recent results have shown that QC approaches for planar beam lattices experiencing in-plane and out-of-plane deformation require higherorder interpolation. Higher-order QC frameworks are scarce nevertheless. In this contribution, the possibilities of a second-order and third-order QC framework are investigated for an elastoplastic spring lattice. The higher-order QC frameworks are compared to the results of the direct lattice computations and to those of a linear QC scheme. Examples are chosen so that both a macroscale and a microscale quantity influences the results. The two multiscale examples focused on are (i) macroscopically prescribed uniaxial deformation and (ii) macroscopically prescribed pure bending. Furthermore, the examples include an individual inclusion in a large lattice and hence, are concurrent in nature.

Copyright line will be provided by the publisher

\section{Introduction}

The mechanical behaviour of fibrous materials is governed by the interplay of fibres at small length scales. Lattice models in which springs and beams are used to represent individual fibre segments and yarn segments can be used to investigate fibrous materials. Examples are the lattice models of [1-4] for (electronic) textiles, those of [5-8] for paper materials, those of $[9,10]$ for fibre glass materials and those of $[11,12]$ for scaffolds for tissue engineering. The simplicity to incorporate fracture probably forms an important reason for the frequent use of lattice models, which has also led to their use for heterogeneous materials [13-15]. Two other advantages of the use of lattice models for fibrous materials are the ease of incorporating large

\footnotetext{
* Corresponding author E-mail: lars.beex@uni.lu
} 
fibre re-orientations [1,2,4], which is not trivial to include in continuum descriptions [16], and the possibility of modelling fibre-to-fibre bond failure [5-7].

A disadvantage of the use of lattice models is their prohibitive computational cost for industrially relevant (macroscale) computations. To overcome this drawback, a number of multiscale approaches can be applied which homogenise or coarse-grain the lattice model. One of those approaches is the quasicontinuum (QC) method [17] which has a specific combination of advantages that makes the approach especially convenient for lattice models of fibrous materials.

First, the QC method is a concurrent multiscale method. This means that it fully resolves the lattice model in regions of interest and coarse-grains the lattice model in regions of less interest. In this way, individual lattice events can be captured by the fully resolved region, whilst a significant computational gain is made in the remaining domain where coarse-graining is applied. Typically the coarse-graining region is much larger than the fully resolved region. The coupling between fully resolved regions and coarse-grained regions can be formulated in a straightforward manner by using kinematics for lattice models governed by nearest neighbour interactions.

Second, the QC method uses the lattice model in the entire modelling domain and hence, no associated constitutive model needs to be formulated and calibrated. This is especially an advantage for lattice models including large fibre/yarn re-orientations and/or fibre/yarn slippage as these mechanics are not straightforward to incorporate in constitutive descriptions.

A final advantage mentioned here is that QC approaches can be formulated in an equationfree manner [18]. This means that no macroscale quantities (e.g. the macroscale stiffness tensor) need to be formulated and coupled to small-scale quantities. Consequently, QC approaches can be simple to implement. Note that not all QC approaches are equation-free; see e.g. [19-21] for equation-free methods and e.g. [17,22] for non-equation-free formulations.

The QC method was originally formulated for (conservative) atomistic lattice models [17] and it has so far mostly been used for these [23-26]. The recent work of [27] on a (conservative) lattice for human erythrocyte membranes forms one of the few exceptions. Recently however, a virtual-power-based QC method [19] was constructed that allows it to be used for non-conservative lattice models (i.e. including dissipation). As non-atomistic lattice models, e.g. those for fibrous materials, often include dissipation, QC approaches can now be applied to a wide variety of lattice models. This has for instance been shown for a lattice model that includes bond failure and subsequent fibre sliding [20] and an elastoplastic lattice model for an electronic textile [21]. In light of applying QC approaches to structural lattice models, recently a QC approach was also formulated for planar beam lattices undergoing in-plane and out-of-plane deformation [28]. It was shown that for these planar beam lattices higher-order interpolation is required, whereas normally linear interpolation is used in QC approaches.

The aim of the current contribution is to investigate the use of higher-order interpolation for lattices in which the interactions use an internally linear interpolation, whereas the beam interactions of the beam lattices in [28] use an internally higher-order interpolation (Hermite interpolation to be exact). The study of [29] also deals with higher-order interpolation for (conservative atomistic) lattices with interactions using internally linear interactions, but focuses on remeshing. In [29] it is observed that a higher-order approach (using variable-node elements) needs less remeshing iterations than a standard linear approach (using tetrahedra) for a given accuracy. This is the main reason according to [29] that the higher-order approach is faster for the same accuracy. In the current contribution remeshing is not of interest. 
Furthermore, the focus is on lattices (with interactions using internally linear interpolation) undergoing large global and local deformations, in contrast to the lattices of [29] undergoing only large local deformations. The question this contribution effectively aims to answer is if higher-order interpolations provide an increased accuracy for lattices undergoing large deformations compared to linear interpolations (for a given computational gain).

The lattice of interest in this study is an elastoplastic spring/truss lattice. As dissipation is included, a standard QC method cannot be used and the virtual-power-based QC variant [19] is adopted. The two multiscale examples that are used to evaluate the different higher-order QC methods focus on uniaxial deformation and pure bending. They are used to investigate which degree of interpolation (1, 2 or 3 ) is most accurate for a fixed computational gain. For this purpose, the QC results are compared to the direct lattice computations (DLCs). Numerical examples are chosen in which the results are influenced by both macroscopic and microscale properties.

The outline of this paper is as follows. In the next section, the elastoplastic truss network is described on the basis of a standard Coleman-Noll procedure. Subsequently, the QC approximation of this lattice model is detailed and clearly distinguishes between the two reduction steps proposed in the QC method: (i) interpolation and (ii) summation/sampling. Afterwards, the descriptions of the two numerical examples are considered in which uniaxial deformation and pure bending are prescribed. Subsequently, the results are presented and finally, conclusions are presented.

\section{Lattice model}

The lattice model of interest in this work is a two-dimensional (2D) X-braced lattice (see Fig. 1). The lattice interactions are formed by elastoplastic springs (i.e. trusses). This is a rather general lattice, as this contribution does not focus on a particular material, although a similar lattice was used in [21] to study an electronic textile. The governing equations of this lattice are recalled in this section for convenience. First, the general thermodynamical setting based on the Coleman-Noll procedure [30] is described. Second, the formulations of the stored energy and dissipation potential of a single elastoplastic lattice interaction are presented. Third, the linearisation of the governing equations is given and the two causes of the prohibitive computational cost associated with using large lattices are presented.

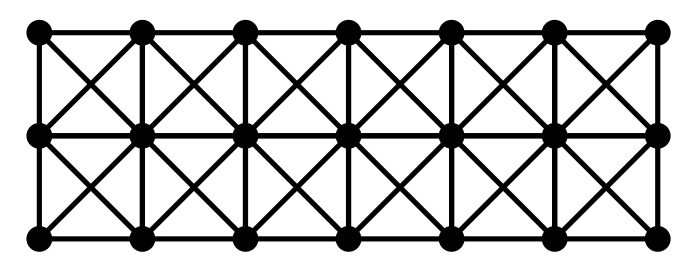

Fig. 1 The X-braced lattice of interest. Black circles are lattice nodes and the black lines represent the lattice interactions, i.e. the elastoplastic springs/trusses. 


\subsection{Coleman-Noll procedure}

The 2D lattice model consists of $b$ lattice interactions and $n$ lattice nodes. These are stored in index sets $B=\{1, \ldots, b\}$ and $N=\{1, \ldots, n\}$, respectively. Hence, the lattice model requires $2 n$ kinematic variables which are the displacement components of the $n$ lattice nodes. They are stored in matrix column $\mathbf{u}$. As the lattice contains elastoplastic interactions, each lattice interaction includes one dissipation mechanism. Consequently, $b$ history variables are required to describe the total dissipation in the lattice. These are stored in matrix column $\mathbf{z}$.

The virtual power statement of the lattice can be expressed as follows:

$$
\dot{\mathbf{u}}^{T} \mathbf{f}=\dot{\mathbf{u}}^{T} \mathbf{g} \quad \forall \dot{\mathbf{u}}
$$

where $\left({ }^{*}\right)$ denotes the time-derivative. Column matrices $\mathbf{f}$ and $\mathbf{g}$ of length $2 n$ represent the internal and external force components, respectively. The left hand side of Eq. (1) represents the virtual internal power, $P$, and the right hand side the virtual external power.

The stored energy of the entire lattice, $E$, depends both on the kinematic variables $\mathbf{u}$ and the history variables $\mathbf{z}$. The stored energy of the lattice model can be expressed as the sum of contributions of individual lattice interactions, $E_{i}$ :

$$
E(\mathbf{u}, \mathbf{z})=\sum_{i=1}^{b} E_{i}(\mathbf{u}, \mathbf{z}) .
$$

The rate of the total stored energy is then determined using the chain rule:

$$
\dot{E}=\dot{\mathbf{u}}^{T} \frac{\partial E}{\partial \mathbf{u}}+\dot{\mathbf{z}}^{T} \frac{\partial E}{\partial \mathbf{z}}
$$

The energy delivered to the lattice per unit of time $(P)$ is either stored or dissipated internally per unit of time:

$$
P=\dot{E}+\dot{D}
$$

where $\dot{D}$ is the rate of dissipation. Substituting Eqs. (1) and (3) in Eq. (4) provides us with the rate of dissipation:

$$
\dot{D}=\dot{\mathbf{u}}^{T}\left(\mathbf{f}-\frac{\partial E}{\partial \mathbf{u}}\right)-\dot{\mathbf{z}}^{T} \frac{\partial E}{\partial \mathbf{z}} \geq 0
$$

clearly expressing that dissipation is not allowed to decrease. Furthermore, in the ColemanNoll procedure [30] dissipation is not allowed to increase if the history variables $\mathbf{z}$ remain constant $(\dot{\mathbf{z}}=\mathbf{0})$. To ensure that this is indeed the case, the following expression is required for the internal forces $\mathbf{f}$ :

$$
\mathbf{f}=\frac{\partial E}{\partial \mathbf{u}}
$$


Using this in Eq. (5), leads to:

$$
\dot{D}=\dot{\mathbf{z}}^{T} \mathbf{f}^{z} \geq 0 \quad \text { with } \quad \mathbf{f}^{z}=-\frac{\partial E}{\partial \mathbf{z}},
$$

where $\mathbf{f}^{z}$ are referred to as the dissipation forces.

The only remaining formulations are those of the stored energy of an individual lattice interaction $\left(E_{i}\right)$ and a dissipation potential for the lattice, $\Phi$, which takes the condition of Eq. (7) into account. The dissipation potential of the lattice can, like the stored energy, be expressed in terms of contributions of individual dissipation mechanisms. As the lattice of interest has one dissipation mechanism per lattice interaction (i.e. spring or truss), $\Phi_{i}$, this can be formulated as:

$$
\Phi(\mathbf{u}, \mathbf{z})=\sum_{i=1}^{b} \Phi_{i}(\mathbf{u}, \mathbf{z})
$$

\subsection{Elastoplastic spring/truss interactions}

In this subsection the remaining expressions of the stored energy and the dissipation potential of an individual elastoplastic spring interaction (i.e. $E_{i}$ and $\Phi_{i}$ ) are presented. These expressions are not specific for the Coleman-Noll procedure, as they dependent amongst others on which type of dissipation mechanism one is interested. However, they satisfy the assumptions introduced in the Coleman-Noll procedure.

The total axial strain of the $i^{\text {th }}$ lattice interaction, $\epsilon_{i}$, is split into an elastic strain, $\epsilon_{i}^{e}$, and a plastic strain, $\epsilon_{i}^{p}$, as follows:

$$
\epsilon_{i}=\epsilon_{i}^{e}+\epsilon_{i}^{p}
$$

The total axial strain can be expressed as:

$$
\epsilon_{i}=\frac{L_{i}-L_{i}^{0}}{L_{i}^{0}} \quad \begin{gathered}
\text { with } \quad L_{i}=\left|\vec{X}_{l}+\vec{u}_{l}-\vec{X}_{k}-\vec{u}_{k}\right| \\
L_{i}^{0}=\left|\vec{X}_{l}-\vec{X}_{k}\right|,
\end{gathered}
$$

where the subscripts $k$ and $l$ refer to the two lattice nodes connected to lattice interaction $i$. Furthermore, $\vec{X}$ and $\vec{u}$ are the original location vector and the displacement vector of a lattice node, respectively. Hence, the components of $\vec{u}_{k}$ and $\vec{u}_{l}$ are present in $\mathbf{u}$. Furthermore, the axial plastic strain of lattice interaction $i$ is set equal to the $i^{\text {th }}$ history variable and so we have $\epsilon_{i}^{p}=z_{i}$. After inserting this together with Eq. (10) in Eq. (9), the following expression for the elastic strain of the $i^{\text {th }}$ lattice interaction is obtained:

$$
\epsilon_{i}^{e}=\frac{\left|\vec{X}_{l}+\vec{u}_{l}-\vec{X}_{k}-\vec{u}_{k}\right|}{\left|\vec{X}_{l}-\vec{X}_{k}\right|}-1-z_{i}
$$


which is needed for the stored energy of lattice interaction $i$ :

$$
E_{i}=\frac{1}{2} \Upsilon_{i} A_{i}^{0} L_{i}^{0}\left(\epsilon_{i}^{e}\right)^{2}
$$

where $\Upsilon_{i}$ and $A_{i}^{0}$ denote the Young's modulus and the original cross-sectional area of lattice interaction $i$, respectively. ()$^{2}$ is used to indicate to the power of 2.

The dissipation potential of lattice interaction $i$ takes the standard form for a 1D system with isotropic hardening of:

$$
\Phi_{i}=\left|F_{i}^{z}\right|-F^{y}\left(\xi_{i}\right) \leq 0,
$$

where $F^{y}\left(\xi_{i}\right)$ is the yield function; for clarity, $F_{i}^{z}=-\frac{\partial E}{\partial z_{i}}=-\frac{\partial E_{i}}{\partial z_{i}}$. History variable $\xi_{i}$ (stored in column matrix $\boldsymbol{\xi}$ for all interactions) is introduced as follows:

$$
\dot{z}_{i}=\dot{\xi}_{i} \frac{\partial \Phi_{i}}{\partial F_{i}^{z}}=\dot{\xi}_{i} \operatorname{sgn}\left(F_{i}^{z}\right) .
$$

Consequently, $\xi_{i}$ is the effective (i.e. equivalent or cumulative) plastic strain of the $i^{\text {th }}$ lattice interaction. To guarantee that dissipation cannot decrease, the following Karush-Kuhn-Tucker (KKT) relations must hold for all interactions:

$$
\dot{\xi}_{i} \geq 0 \quad \Phi_{i} \leq 0 \quad \dot{\xi}_{i} \Phi_{i}=0 .
$$

The following yield function is adopted in this contribution, defined for interaction $i$ :

$$
F_{i}^{y}\left(\xi_{i}\right)=A_{i}^{0} L_{i}^{0} \sigma_{i}^{0}\left(1+\alpha_{i}\left(\xi_{i}\right)^{\beta_{i}}\right),
$$

where $\sigma_{i}^{0}$ denotes the initial yield stress of interaction $i$ and $\alpha_{i}$ and $\beta_{i}$ are its hardening parameters.

\subsection{Problems with solving the governing equations of macroscale computa- tions}

The governing equations of the lattice model are derived by equilibrating the forces in Eq. (1), whilst ensuring that the KKT conditions of Eq. (15) are met for each lattice interaction. The equations resulting from Eq. (1) are nonlinear because of the material behaviour and because large rotations are included. Hence, a Newton-Raphson procedure is adopted in order to linearise and solve the system. This can be expressed as follows:

$$
\mathbf{f}\left(\mathbf{u}^{*}, \mathbf{z}^{*}\right)+\mathbf{K}\left(\mathbf{u}^{*}, \mathbf{z}^{*}\right) d \mathbf{u}=\mathbf{g},
$$

where $\mathbf{u}^{*}$ and $\mathbf{z}^{*}$ are the kinematic and history variables of the previous iteration, respectively, and $d \mathbf{u}$ are the corrections to $\mathbf{u}^{*}$. The history variables $\mathbf{z}$ in the current iteration are found by 
ensuring that the KKT conditions are met. This is most often performed individually for each component of $\mathbf{z}$. In Eq. (17), $\mathbf{K}$ is the stiffness matrix which is computed by taking the derivatives of $\mathbf{f}$ with respect to the kinematic variables, i.e. the second order derivatives of $E$ with respect to the kinematic variables. Similarly to $\mathbf{f}$, all lattice interactions need to be visited to construct it. The contribution of the $i^{\text {th }}$ lattice interaction to $\mathbf{f}$ and $\mathbf{K}$ (i.e. $\mathbf{f}_{i}$ and $\mathbf{K}_{i}$ ) can be expressed as follows:

$$
\begin{aligned}
& \mathbf{f}=\frac{\partial E}{\partial \mathbf{u}}=\sum_{i=1}^{b} \frac{\partial E_{i}}{\partial \mathbf{u}}=\sum_{i=1}^{b} \mathbf{f}_{i}, \\
& \mathbf{K}=\frac{\partial}{\partial \mathbf{u}}\left(\frac{\partial E}{\partial \mathbf{u}}\right)=\sum_{i=1}^{b} \frac{\partial}{\partial \mathbf{u}}\left(\frac{\partial E_{i}}{\partial \mathbf{u}}\right)=\sum_{i=1}^{b} \mathbf{K}_{i} .
\end{aligned}
$$

After the Dirichlet boundary conditions (BCs) are incorporated in $\mathbf{u}$ and the Neumann BCs in $\mathbf{f}$, the system needs to be partitioned in a standard manner before it can be solved.

Note that the $p^{\text {th }}$ component of $\mathbf{f}_{i}$ and the $p q^{\text {th }}$ component of $\mathbf{K}_{i}$ must be computed as follows:

$$
\begin{aligned}
& \left(f_{i}\right)_{p}=\frac{\partial E_{i}}{\partial u_{p}}, \\
& \left(K_{i}\right)_{p q}=\frac{\partial}{\partial u_{q}}\left(\frac{\partial E_{i}}{\partial u_{p}}\right),
\end{aligned}
$$

where $p$ and $q$ run over all components of $\mathbf{u}$. Note also that if $p$ and $q$ run over all components of $\mathbf{u}$ in a QC method, this results in an energetically-consistent method (as was made clear in $[19,31,32])$, in contrast to a force-based QC method such as the one of [33].

The fact that all $b$ lattice interactions must be visited to construct the governing equations (i.e. to assemble $\mathbf{f}$ and $\mathbf{K}$ ) is one of the two causes of the prohibitive computational cost of macroscale computations for lattice models in which each interaction is used to represent a fibre segment or yarn segment. Second, the number of $2 n$ equations for macroscale computations is large.

\section{Virtual-power-based Quasicontinuum method}

The multiscale QC method [17] proposes two remedies to overcome the prohibitive computational cost of macroscale lattice computations. First, interpolation of the kinematic variables is introduced to reduce the degrees of freedom (i.e. the displacement components) so that a significantly smaller system needs to be solved. Second, only a small number of lattice interactions is used to sample the governing equations, i.e. $\mathbf{f}$ and $\mathbf{K}$, instead of visiting all $b$ interactions. This latter reduction step is often referred to as summation or sampling.

Both reduction steps proposed in the QC method involve an approximation. Interpolation of the kinematic variables means that the solution space is reduced and hence, an error due to 
interpolation occurs. Second, the use of only a small number of lattice interactions to sample the contributions of all interactions means that an error due to sampling may occur. In Fig. 2 these the reduction steps and associated errors are illustrated. The crux of the method is to apply both reduction steps such that the total error is insignificant.

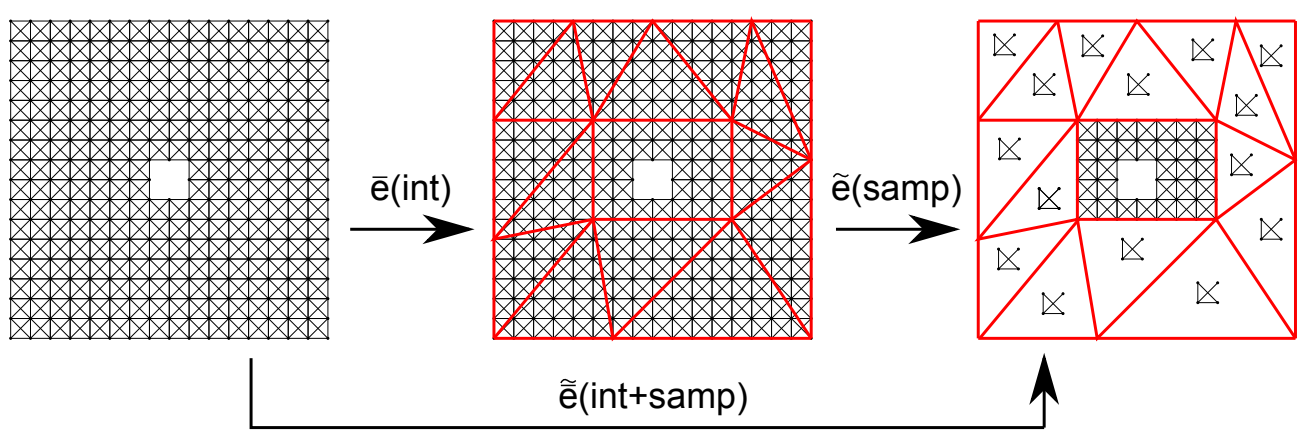

Fig. 2 The two reduction steps of the QC method: interpolation and summation/sampling. Left: the full lattice model, centre: the interpolated lattice model, and right: the interpolated and sampled lattice model (i.e. the full QC model). The error due to interpolation is indicated as $\bar{e}$, the error due to sampling/summation as $\widetilde{e}$ and the error due to interpolation and summation as $\widetilde{\bar{e}}$.

\subsection{Interpolation}

In the QC method, interpolation of the displacement components of the lattice nodes generally makes use of finite element (FE) shape functions. To this purpose, an FE triangulation is superimposed on the lattice model and the FE shape functions are evaluated at the lattice nodes. The shape function evaluations are stored in a condensation matrix, $\Psi$. The condensation matrix is used to express the displacement components of all $n$ lattice nodes $\mathbf{u}$ in terms of the small number of displacement components of the triangle nodes $\mathbf{u}^{r}$ as follows:

$$
\mathbf{u} \approx \overline{\mathbf{u}}=\mathbf{\Psi} \mathbf{u}^{r},
$$

where $\overline{\mathbf{u}}$ represents the column matrix with the interpolated displacement components of all $n$ lattice nodes. Condensation matrix $\boldsymbol{\Psi}$ is thus of size $2 n \times 2 r$ for a $2 \mathrm{D}$ lattice, where $r$ denotes the number of nodes of the interpolated system. If Eq. (22) is inserted in Eq. (1) and the result is linearised, the equations of the condensed/interpolated force equilibrium read:

$$
\Psi^{T} \mathbf{f}+\Psi^{T} \mathbf{K} \Psi d \mathbf{u}^{r}=\Psi^{T} \mathbf{g},
$$

in contrast to Eq. (17). Above, $\Psi^{T} \mathbf{f}$ and $\boldsymbol{\Psi}^{T} \mathbf{K} \Psi$ can be recognised as the interpolated internal force column, $\overline{\mathbf{f}}$, and the interpolated stiffness matrix $\overline{\mathbf{K}}$, respectively. The number of equations of the interpolated system is $2 r$, which is significantly smaller than the full set of equations if $r \ll n$.

Traditionally in QC approaches the $r$ nodes of the interpolated system are triangle nodes which are located on top of a small number of lattice nodes. The small number of lattice 
nodes that correspond to the triangle nodes are often referred to as repnodes (or repatoms when the lattice of interest is an atomistic lattice). In regions of interest triangle nodes are placed on top of all lattice nodes so that the lattice model is fully resolved. This makes the QC method a concurrent multiscale approach, as in small regions of interest the lattice model is fully included but in the remaining domain it is coarse-grained (i.e. interpolated and sampled, see also the next subsection). Furthermore, conforming triangulations are generally used to achieve a smooth transition from fully resolved domains to coarse-grained domains (see e.g. [17, 19-26,31-33]).

In the QC approaches in this contribution, triangulations are only used in coarse domains (as schematically illustrated in Fig. 2). This is done for two reasons. First, our previous study [32] has indicated that refining the triangulation in the coarse domain has little influence on the accuracy in the fully resolved domain, i.e. the domain in which one generally desires a high accuracy. The size of the fully resolved domain on the other hand has a large influence on the accuracy. Hence, an approach that delivers a high accuracy in the fully resolved domain while saving as much computational effort as possible, requires relatively large fully resolved regions in combination with large triangles elsewhere. The consequence of this observation would be only to use large triangles in the coarse domain, coupling this in a non-conforming manner to the fully resolved lattice model in the domain of high interest.

Second, for QC approaches with higher-order interpolation and conforming triangulations a numerical issue occurs concerning the triangles in the transition zone between the fully resolved domains and the coarse-grained domains. In [28] it was observed that the triangles in the transition zone in QC approaches with higher-order interpolation have too many triangle nodes, i.e. too many degrees of freedom, than are governed by underlying lattice. Obviously, this is also the case for the triangles in the fully resolved domain, but as the size and dimensions of those triangles are known, this numerical issue can straightforwardly be overcome by locally reducing the order of the interpolation. However, this cannot be done, a priori, for the triangles in the transition zone [28].

A simple remedy to avoid this numerical issue, also exploited in this contribution, is to use relatively large triangles in the coarse-grained domain and couple them kinematically in a non-conforming manner to the fully resolved lattice model in the domain of interest [28]. Hence, in this work no transition regime is present at all. Furthermore, three QC schemes are proposed in this contribution: one that uses linear interpolation in the coarse domain, one with quadratic interpolation in the coarse domain and one with cubic interpolation in the coarse domain. In each QC approach, only one type of interpolation is present in the coarse domain. The coarse domain in each QC framework is kinematically coupled to the fully resolved domain and hence, no transition regime is present.

The advantages of using large triangles in the coarse domain and coupling them directly to fully resolved lattice models are thus that (i) no numerical issues occur if higher-order interpolation is used and (ii) a larger computational gain is made for a similar accuracy in the fully resolved regions, compared to conforming triangulations. The disadvantage is that a coupling procedure has to be formulated and implemented, but for lattice models with only nearest neighbour interactions straightforward kinematic coupling is sufficient (also employed in this contribution). Furthermore, note that in the current study, triangle nodes in the coarsegrained domain are not located on top of lattice nodes. 
Although the number of equations is significantly reduced by the introduction of interpolation, the disadvantage that all $b$ lattice interactions need to be visited to construct Eq. (23) remains. The expressions of $\overline{\mathbf{f}}$ and $\overline{\mathbf{K}}$ now read as follows:

$$
\begin{gathered}
\overline{\mathbf{f}}=\frac{\partial \bar{E}}{\partial \mathbf{u}^{r}}=\boldsymbol{\Psi}^{T} \frac{\partial E}{\partial \mathbf{u}}=\boldsymbol{\Psi}^{T} \sum_{i=1}^{b} \frac{\partial E_{i}}{\partial \mathbf{u}}=\boldsymbol{\Psi}^{T} \sum_{i=1}^{b} \mathbf{f}_{i} \\
\overline{\mathbf{K}}=\frac{\partial}{\partial \mathbf{u}^{r}}\left(\frac{\partial \bar{E}}{\partial \mathbf{u}^{r}}\right)=\boldsymbol{\Psi}^{T} \frac{\partial}{\partial \mathbf{u}}\left(\frac{\partial E}{\partial \mathbf{u}}\right) \boldsymbol{\Psi}=\boldsymbol{\Psi}^{T} \sum_{i=1}^{b} \frac{\partial}{\partial \mathbf{u}}\left(\frac{\partial E_{i}}{\partial \mathbf{u}}\right) \boldsymbol{\Psi}=\boldsymbol{\Psi}^{T} \sum_{i=1}^{b} \mathbf{K}_{i} \boldsymbol{\Psi}
\end{gathered}
$$

Also the KKT conditions (Eq. (15)) still have to be met for all $b$ lattice interactions.

Note that the components of $\mathbf{f}_{i}$ and $\mathbf{K}_{i}$ are still computed according to Eq. (20) and Eq. (21), respectively.

\subsection{Sampling/Summation}

To overcome the computational effort of visiting all $b$ lattice interactions to construct the governing equations of the interpolated system, i.e. $\overline{\mathbf{f}}$ and $\overline{\mathbf{K}}$, only a small number of $s$ lattice interactions $(s \ll m)$ are selected in QC methods to sample the contributions of all $b$ lattice interactions. Each of the $s$ sampling interactions represents the contributions of $w_{i}$ lattice interactions. Consequently, the force column and stiffness matrix of the interpolated and sampled system (i.e. of the QC system), $\widetilde{\overline{\mathbf{f}}}$ and $\widetilde{\bar{K}}$, are determined as follows:

$$
\begin{aligned}
\widetilde{\mathbf{f}} & =\Psi^{T} \sum_{i \in S} w_{i} \frac{\partial E_{i}}{\partial \mathbf{u}}=\Psi^{T} \sum_{i \in S} w_{i} \mathbf{f}_{i} \\
\widetilde{\mathbf{K}} & =\Psi^{T} \sum_{i \in S} w_{i} \frac{\partial}{\partial \mathbf{u}}\left(\frac{\partial E_{i}}{\partial \mathbf{u}}\right) \boldsymbol{\Psi}=\boldsymbol{\Psi}^{T} \sum_{i \in S} w_{i} \mathbf{K}_{i} \boldsymbol{\Psi},
\end{aligned}
$$

where $S$ is the set of sampling interactions. The components of $\mathbf{f}_{i}$ and $\mathbf{K}_{i}$ are still computed using Eqs. (20) and (21), respectively.

The degrees of freedom of the interpolated system, stored in $\mathbf{u}^{r}$, remain unaffected by the sampling procedure and hence, the governing equations (23) are still valid. The only difference introduced by sampling is the manner in which they are constructed, cf. Eqs. (26) and (27).

The sampling procedure is based on the assumption that the stored energy and dissipation of the interpolated system remain unaffected by sampling:

$$
\bar{E} \approx \widetilde{\bar{E}}=\sum_{i \in S} w_{i} E_{i},
$$




$$
\bar{D} \approx \widetilde{\bar{D}}=\sum_{i \in S} w_{i} \int-\dot{z}_{i} \frac{\partial E_{i}}{\partial z_{i}} d t
$$

To this purpose, the dissipation potential of sampling interaction $i$ is reformulated as:

$$
\widetilde{\bar{\Phi}}_{i}=\left|\widetilde{\overline{F_{i}^{z}}}\right|-w_{i} F^{y}\left(\xi_{i}\right) \leq 0
$$

in contrast to Eq. (13). Note that the term $\widetilde{\overline{F_{i}^{z}}}$ in Eq. (30) results in $-w_{i} \frac{\partial E_{i}}{\partial z_{i}}$, in which the effect of sampling can clearly be observed.

In this contribution, the sampling procedure is based on that of [34] which is amongst others inspired by [35] and [36]. In the sampling procedure, the $s$ sampling interactions are not selected from the existing set of lattice interactions, except in fully resolved domains. In fully resolved domains all lattice interactions form sampling interactions that only represent themselves (i.e. $w_{i}=1$ ).

In the coarse domain however, each type of sampling interaction is located on top of a Gauss quadrature point (GQP). As the lattice of interest is an X-braced lattice, four types of sampling interactions are located on top of each GQP. The weight factor $w_{i}$ of sampling interaction $i$ is determined according to:

$$
w_{i}=w_{g q p} l_{t r} \quad \text { with } \quad l_{t r}=\frac{A_{t r} l_{u c}}{A_{u c}},
$$

where $w_{g q p}$ are the standard weights of GQPs in FE technology (see [28] and [37]) and $l_{t r}$ is the number of lattice interactions of a particular type per triangle. The latter is in this contribution straightforwardly computed using the area of a triangle, $A_{t r}$, the area of a unit cell, $A_{u c}$ and the number of lattice interactions of a particular type per unit cell, $l_{u c}$. As a result of Eq. (31), the four sampling interactions per GQP each have the same weight factor $w_{i}$ for the X-braced lattice of interest.

For the three types of interpolations investigated in this contribution (linear, quadratic and cubic), one, three and six GQPs are used per triangle, respectively. Although for standard FE technology it is sufficient to use four GQPs per triangle for cubic interpolation, this is not sufficient if lattices are interpolated and sampled [28].

\section{Descriptions of the numerical examples}

In this contribution two multiscale examples are considered to evaluate the three QC approaches with (i) linear, (ii) quadratic and (iii) cubic interpolations. The QC approaches include a fully resolved region in which a stiff domain is incorporated as to include the concurrent aspect of the method. The QC results are compared to those of the direct lattice computations (DLCs). Macroscopic uniaxial deformation is considered in the first example and macroscopic pure bending in the second.

The examples are chosen in which a global quantity as well as a microscale quantity influences the results. In the first example the sum of the horizontal reaction forces on one 
boundary is determined as a function of the prescribed displacement (i.e. the global quantity). Furthermore, it is assumed that when one of the lattice interactions reaches a strain of $15 \%$ (governed by the microscale), fracture occurs, and hence, the subsequent global response is of no interest. In the example of pure bending, instead of the horizontal reaction forces, the total moment is computed for the prescribed angle (i.e. the global quantity). Also in this case, the comparison is made until one of the lattice interactions reaches a strain of $15 \%$ (i.e. the microscale quantity). A strain of $15 \%$ is chosen as this strain occurs at large deformations and hence, requires a large deformation framework (as described in Subsection 2.2). On the other hand, it is sufficiently small so that a relatively small number of increments suffices.

The geometrical and material parameters of the $\mathrm{X}$-braced lattice model are given in Table 1. All geometrical and material parameters of the lattice interactions in the stiff region are the same, except that the Young's modulus $\left(\Upsilon_{i}\right.$ in Eq. (12)) is 100 times larger and an infinitely large initial yield stress $\left(\sigma_{i}^{0}\right.$ in Eq. (16)) is used to prevent plastic yielding. The modelling domain is in both examples $200 \times 100$ lattice spacings in the horizontal and vertical directions, respectively. Hence, the DLC involves 40,602 kinematic variables and 80,300 lattice interactions. Furthermore, the stiff region in both examples is of size $8 \times 8$ lattice spacings.

Table 1 Geometrical and material parameters of the lattice interactions in the 2D X-braced lattice model. $L^{0, x}$ and $L^{0, y}$ refer the lattice spacing in horizontal and vertical direction, respectively.

\begin{tabular}{|l|l|}
\hline$A^{0}\left[\mathrm{~mm}^{2}\right]$ & 1 \\
\hline$L^{0, x}[\mathrm{~mm}]$ & 1 \\
\hline$L^{0, y}[\mathrm{~mm}]$ & 1 \\
\hline$\Upsilon[G P a]$ & 1 \\
\hline$\sigma^{0}[G P a]$ & 0.01 \\
\hline$\alpha[]$ & 10 \\
\hline$\beta[]$ & 0.5 \\
\hline
\end{tabular}

For both examples, the triangulations of the QC approaches are made such that approximately the same number of sampling interactions is required for each order of interpolation. This ensures that approximately the same computational time is required for each QC computation, regardless of the order of interpolation. As a triangle with linear interpolation requires one GQP (i.e. 4 sampling interactions), a triangle with quadratic interpolation three and one with cubic interpolation six GQPs, a significantly different number of triangles is used for each QC approach (see ahead to Fig. 4). To make sure that the boundary of the fully resolved region has approximately the same number of degrees of freedom in each QC scheme, smaller triangles are used next to the fully resolved region in the QC method with linear interpolation (see again Fig. 4).

\subsection{Uniaxial deformation}

In the numerical example regarding global uniaxial deformation, the stiff region is centred in the modelling domain (see ahead to Fig. 4). In all QC variants the fully resolved domain (including the stiff region) is of size $20 \times 20$ lattice spacings. In the QC framework with linear interpolation 248 triangles are used in the coarse domain compared to 62 and 30 for the 
frameworks with quadratic and cubic interpolation, respectively. For all three QC schemes, this means that the number of sampling interactions is approximately $3 \%$ of that of the DLC. Hence, the QC computations are approximately 33 times as fast as that of the DLC.

Uniaxial deformation up to a horizontal strain of $10 \%$ is prescribed by prohibiting the nodes (lattice nodes in the DLC and triangle nodes in the QC computation) on the left boundary of the modelling domain to displace and prescribing the horizontal displacements of the nodes on the right boundary to displace to $20 \mathrm{~m}$ in 250 increments. The nodes on the right boundary are also prevented from moving in the vertical direction, similar to the nodes on the top and bottom boundaries.

\subsection{Pure bending}

The stiff region in the pure bending example is centred horizontally and the bottom boundary of the stiff region corresponds to the bottom boundary of the modelling domain (see ahead to Fig. 8). In all QC variants the fully resolved region is of size $20 \times 14$ lattice spacings in horizontal and vertical direction, respectively. The coarse domains in the different QC schemes are discretised using 216 (linear), 76 (quadratic) and 38 (cubic) triangles. Consequently, the number of sampling interactions in the three QC frameworks is approximately $2.5 \%$ of that of the DLC. Hence, the QC computations are approximately 40 times faster than the DLC.

The BCs for the pure bending example are based on the work of [38], although a slightly different formulation was found for the ratio between horizontal displacement components and vertical displacement components on the right boundary (see ahead to Eq. (32)). The aim of the BCs is to apply bending such that lateral contraction is free to occur in the configuration presented in Fig. 3.

The BCs on the left edge of the model are as follows. First, the horizontal and vertical displacement components of the center node of the model (lattice node in the DLC and triangle node in the QC computations) at a height of $\frac{1}{2} H \vec{e}^{y}$ are both suppressed (see Fig. 3 for the definition of the symbols). Note that this requires that in the QC triangulations a triangle node is present on the left model edge at a height of $\frac{1}{2} H \vec{e}^{y}$. Furthermore, in order to let contraction take place freely, the vertical displacement components of the remaining nodes on the left model edge are not prescribed (and hence, the Neumann BCs of these nodes are that the vertical reaction forces must be zero), whilst the horizontal displacement components of these nodes are set to zero.

To allow contraction to take place freely on the right side of the model, Dirichlet and Neumann BCs are not sufficient. Instead, the following two relations are prescribed for each node on the right model edge:

$$
\begin{aligned}
& u^{x}-\left(u^{y}-\frac{W}{\tan (\theta)}+\frac{W}{\theta}-\frac{1}{2} H+Y\right) \tan (\theta)=0, \\
& f^{x} \tan (\theta)+f^{y}=0
\end{aligned}
$$

where $f$ refers to a force component of a node on the right model edge and $u$ to a displacement component. The superscripts refer to the directions. Furthermore, $Y$ refers to the vertical location vector component of a node in the original configuration, $W$ to the width of the 
domain, $H$ to the height of the domain and $\theta$ to the applied bending angle (see Fig. 3). Note that the term $-\frac{W}{\tan (\theta)}+\frac{W}{\theta}$ in Eq. (32) does not occur in the first equation of Eq. (10) in [38].

The relations in Eqs. (32) and (33) can be incorporated in the governing equations via Lagrange multipliers (see e.g. [39]), but in this contribution they are incorporated by replacing the horizontal and vertical force equilibria of the (lattice or triangle) each node on the right edge in Eqs. (17) and (23). To ensure that the residual originating from Eq. (32) is of a similar order of magnitude as the other equations, Eq. (32) is multiplied with a factor of $100 \Upsilon$. Pure bending is then prescribed by increasing the bending angle $(\theta)$ in 250 increments to a final value of $20^{\circ}$.

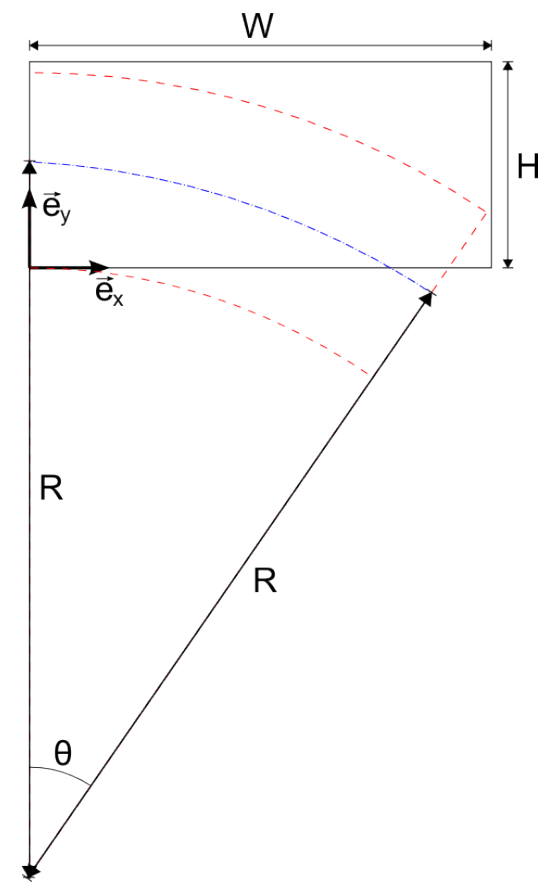

Fig. 3 Schematic deformation of the model during pure bending with an angle $\theta$ and a radius $R$. The original shape is presented using black lines whereas the deformed shape using dashed red lines. The centre line of the model that was originally at a height of $\frac{1}{2} H \vec{e}^{y}$ is shown during pure bending as a dashed-dotted blue line.

The remaining part of this subsection is dedicated to explaining the formulations in Eqs. (32) and (33) that describe free contraction at the right side of the model. Eq. (33) does not require much explanation as it simply describes that the force components of the nodes at the right edge must be zero in the tangential direction during bending (see Fig. 3).

The explanation of Eq. (32) starts with the assumption that the centre line at a height of $\frac{1}{2} H \vec{e}^{y}$ in the original configuration forms a part of a circle without being elongated when pure bending is applied to the model (dashed-dotted blue arc in Fig. 3). Hence, this centre line always remains of length $W$ which leads to the following relation: 


$$
R=\frac{W}{\theta}
$$

Furthermore, the original location vector, $\vec{X}$, of a node on the right edge at a height $Y$ in the initial configuration, its displacement vector, $\vec{u}$, and its location vector in the current configuration, $\vec{x}$, can be formulated as follows:

$$
\begin{aligned}
& \vec{X}=W \vec{e}^{x}+Y \vec{e}^{y}, \\
& \vec{u}=u^{x} \vec{e}^{x}+u^{y} \vec{e}^{y}, \\
& \vec{x}=\vec{X}+\vec{u}=x \vec{e}^{x}+y \vec{e}^{y} .
\end{aligned}
$$

Based on Fig. 3 furthermore, it can be deducted that the relation between the vertical location vector component in the current configuration, $y$, and the horizontal location vector component in the current configuration, $x$, is as follows:

$$
y(x)=\frac{x}{\tan (\theta)}-R+\frac{1}{2} H .
$$

Eq. (32) can then be found by combining Eqs. (34-38).

\section{Results}

In this section the results of the DLC are compared to the results of the three QC schemes. First, the results for uniaxial deformation are considered and subsequently, those for pure bending.

\subsection{Uniaxial deformation}

In Fig. 4 and Fig. 5 the total strains of the lattice/sampling interactions are shown for uniaxial deformation at the increment in which one of them reaches a strain of $15 \%$. It is clearly observable that the stiff region introduces a fluctuation field. Although the fluctuation field reaches as far as the top and bottom boundaries of the model, the most significant part of the field occurs in the vicinity of the stiff region. Hence, the most significant part of the fluctuation field is within the fully resolved regions of the QC computations and it is therefore well captured (Fig. 5).

In Fig. 5 the lattice interactions that reach a total strain of $15 \%$ are shown in black. In all results, the lattice interactions with the highest strain are the horizontal ones next to one of the four corners of the stiff region. As the model is symmetric with respect to the horizontal centre as well as the vertical centre, the decision which of these four interactions reaches $15 \%$ first is in principle determined by numerical truncations and of no importance for the comparison. After all, if only a quarter of the domain were modelled, the same interaction would reach $15 \%$ in the DLC results and QC results. 

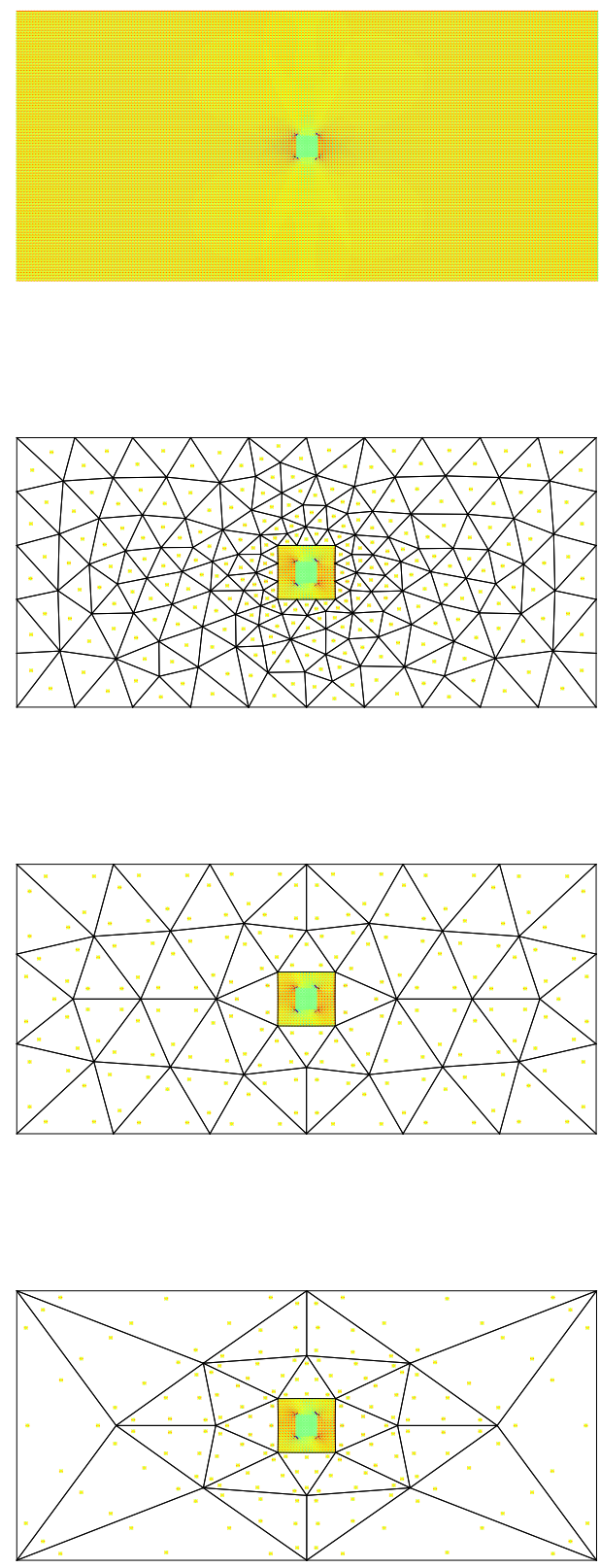

Fig. 4 The total strains of the lattice interactions as predicted by the DLC (top) and of the sampling

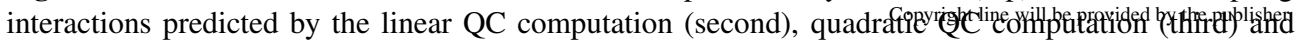
cubic QC computation (fourth) for uniaxial deformation at the increment at which the total strain in one lattice interaction reaches $15 \%$. Note that the colour bar is not symmetric. Triangles are presented in black. 


\begin{tabular}{rr}
-0.02 & 0 \\
\hline
\end{tabular}
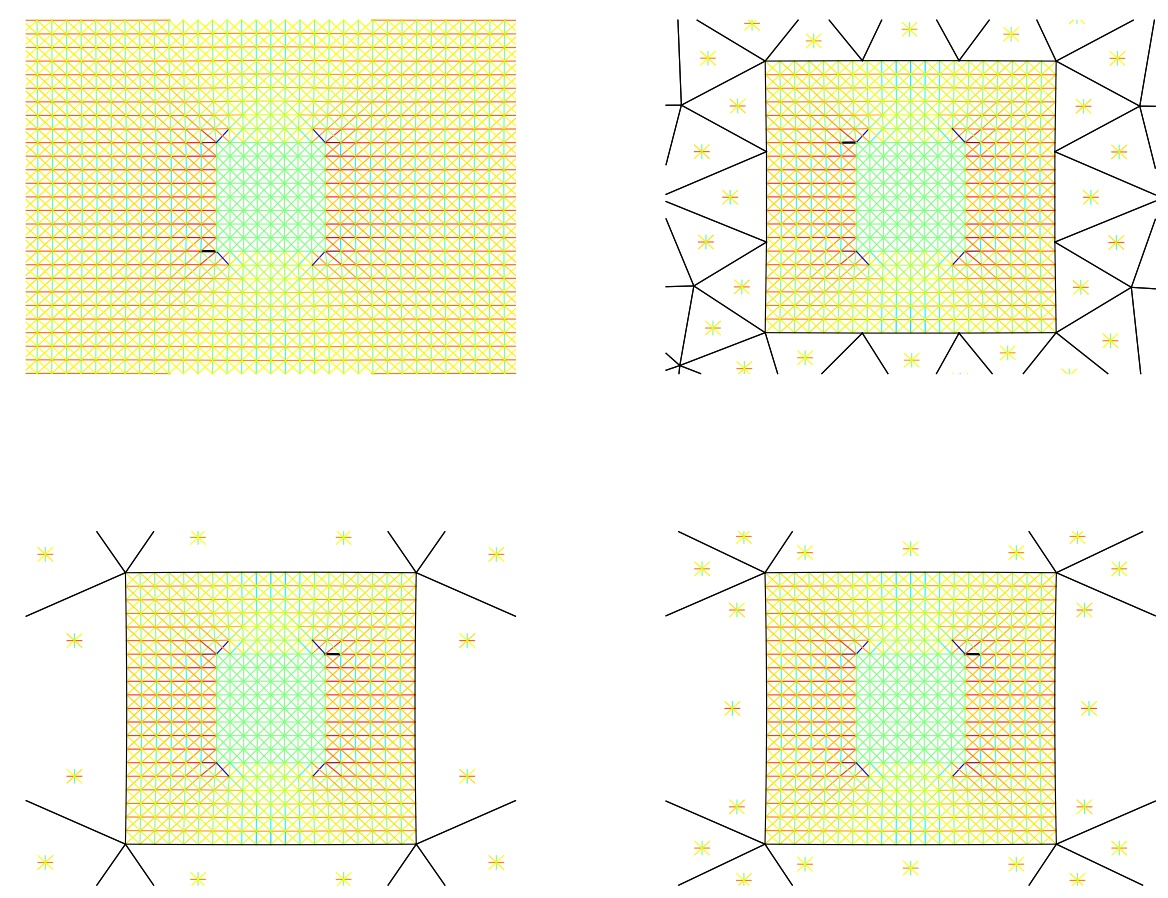

Fig. 5 Enlargements of the stiff region showing the total strains of the lattice interactions as predicted by the DLC (top-left) and of the sampling interactions predicted by the linear QC computation (topright), quadratic QC computation (bottom-left) and cubic QC computation (bottom-right) for uniaxial deformation at the increment at which the total strain in one lattice interaction reaches $15 \%$. The lattice interaction that reaches a total strain of $15 \%$ is shown in black. Note that the colour bar is not symmetric. Triangles are presented in black.

The sum of the horizontal reaction forces at the right boundary is presented as a function of the horizontal displacement of the same boundary in Fig. 6. Fig. 6 clearly demonstrates that the responses predicted by the QC computations all match that of the DLC. Both the initial stiffness and the stiffness in the plastic regime are the same for the DLC and the QC methods. The match of the curves is perfect for all QC schemes, except when the responses are truncated because one of the interactions reaches $15 \%$. In all QC computations one of the interactions clearly reaches $15 \%$ before this occurs in the DLC. This observation is understandable as the lattice models in the fully resolved domains in the QC computations are more constrained than those in the same domain in the DLC. 


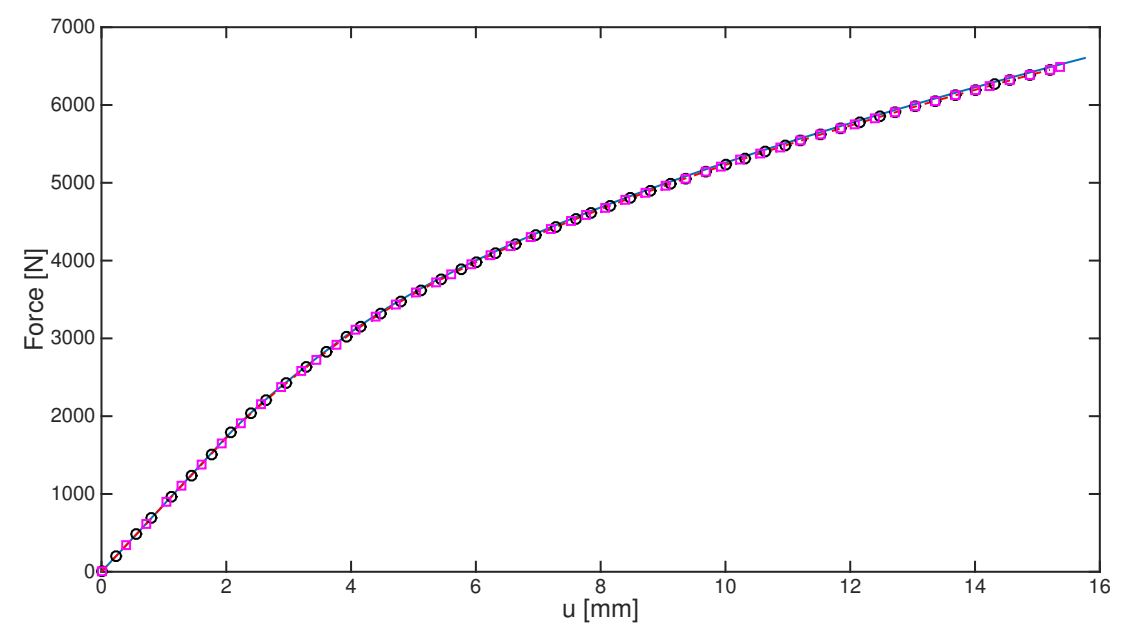

Fig. 6 The force-displacement curves predicted by the DLC (blue, solid), the linear QC computation (red, dashed), the quadratic QC computation (black circles) and the cubic QC computation (magenta squares) for uniaxial deformation until one of the lattice interactions reaches a strain of $15 \%$.

In Fig. 7 the relative errors of the local strains of the horizontal interactions are shown for the quadratic QC approach. The quadratic approach is only shown as an example, similar results are observed for the linear and cubic QC approaches. The relative errors are determined as follows:

$$
e_{i}=\left|\frac{\epsilon_{i}^{Q C}-\epsilon_{i}^{D L C}}{\epsilon_{i}^{D L C}}\right| \cdot 100 \%,
$$

where $e_{i}$ is the relative error of the local strain of horizontal interaction $i, \epsilon_{i}^{Q C}$ the total strain of this interaction predicted by the QC approach and $\epsilon_{i}^{D L C}$ the total strain of this interaction predicted by the DLC. Note that since in the coarse domain the local strains are known only for a small number of sampling interactions in QC computations, the relative errors presented in Fig. 7 are based on post-processed/extrapolated values for all horizontal interactions. In other words, only in the sampling interactions the strains are known (see e.g. Fig. 4) and to determine the local strains of all interactions as presented in Fig. 7, additional post-processing efforts need to be performed.

All the relative errors in Fig. 7 are computed for the quadratic QC approach with the same triangulation as shown in Fig. 4, but with slightly different fully resolved regions - each fully resolved region is increased by $4 \times 4$ lattice spacings. A nice aspect of the presented QC schemes observable in Fig. 7 is that the relative errors of the local strains are smaller in and around the fully resolved region, if the size of the fully resolved region increases. This is in contrast to the results presented for the central summation rule in [19]. However, if the triangle edges at the borders of the fully resolved domain would not be aligned with the lattice 

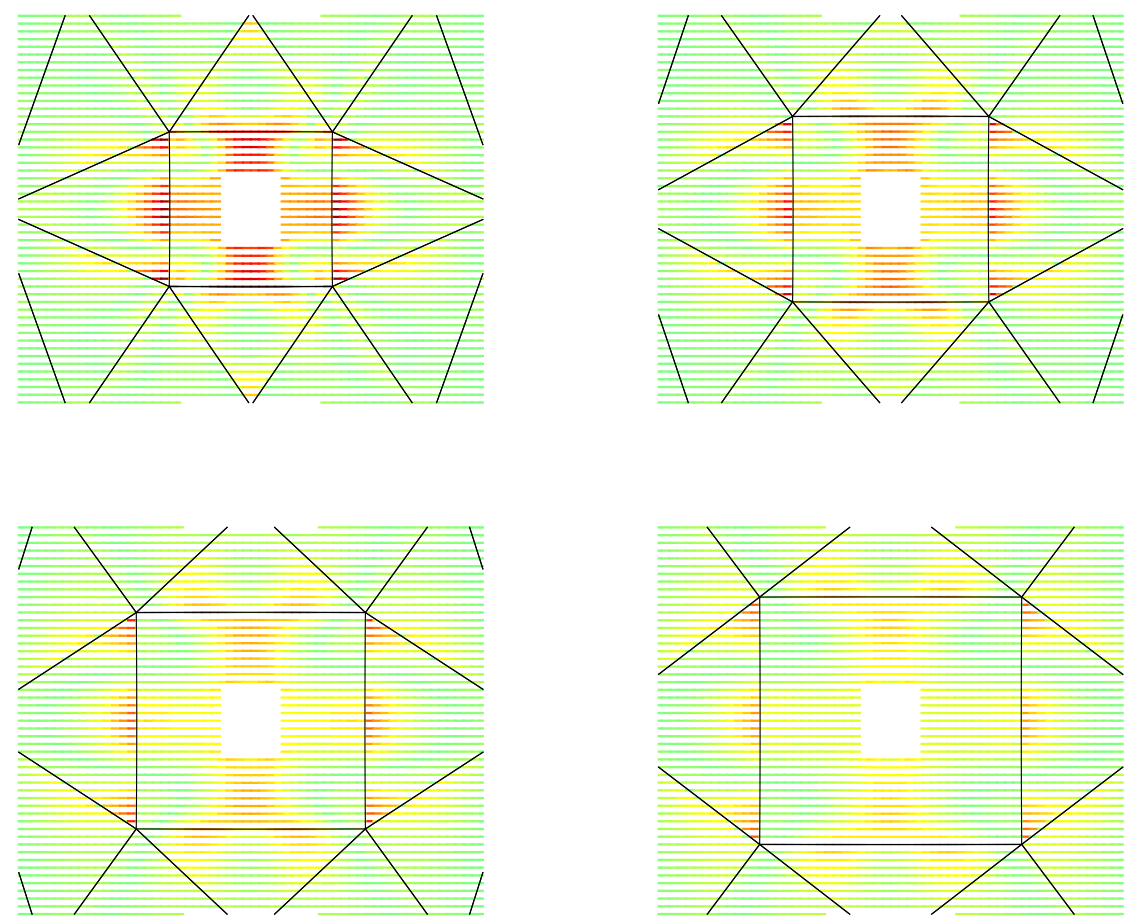

Fig. 7 Enlargements of the stiff region showing the relative errors of the total strains of the horizontal lattice interactions of the quadratic QC framework for the same triangulation but with different sizes of fully resolved domains. The relative errors are determined after increment 150 and hence, at an applied displacement of $12 \mathrm{~m}$. The top-left image shows the relative errors for the reference case (i.e. with a fully resolved region of $20 \times 20$ lattice spacings), the top-right image shows them for a fully resolved region of $24 \times 24$ lattice spacings, the bottom-left image shows them for a fully resolved region of $28 \times 28$ lattice spacings and the bottom-right image shows them for a fully resolved domain of $32 \times 32$ lattice spacings. Triangles are presented in black.

and/or if the lattice would include longer interactions than only nearest-neighbour interactions, these results are very likely to change.

The most important conclusion based on the case of uniaxial deformation is that the global responses predicted by the QC frameworks match the DLC response perfectly, until one lattice interaction fails. In all QC results the first failure of a lattice interaction happens at approximately $95 \%$ of the total global deformation reached in the DLC results. Hence, no particular QC framework is more accurate than any other for the case of uniaxial deformation for a fixed computational gain. 


\subsection{Pure bending}

The total strains of the lattice/sampling interactions predicted by the DLC and QC computations are shown in Fig. 8 and Fig. 9 for macroscopic pure bending at the increment in which one of the interactions reaches 15\%. Based on the top image of Fig. 8 it may be clear that the significant part of the fluctuation field caused by the stiff region in the pure bending example decays over approximately 10 lattice spacings in horizontal direction as well as in vertical direction. The fluctuation field on the left and right side of the stiff inclusion clearly reveals itself as bands crossing each other, starting from the corners of the stiff region. Above the inclusion no bands are observable, but fluctuating strains can nevertheless clearly be observed. The significant part of the fluctuation field that is present in the fully resolved domains of the QC computations seems well predicted by the QC computations, based on Fig. 9.

The QC frameworks are also accurate in terms of which lattice interaction is the first to reach 15\% (see again Fig. 9). In all cases this lattice interaction is either the horizontal one next to the bottom-left corner either the horizontal one next to the bottom-right corner. Analogously to the uniaxial deformation case, it is of no importance which of these two reaches $15 \%$, as the numerical example is in principle symmetric.

The moment applied at the left boundary is presented as a function of the applied angle in Fig. 10. The responses show two stages. During the first stage all interactions only deform elastically, except for some around the stiff domain. From an angle of approximately 3.5\% onwards the lattice interactions at the bottom and top boundaries deform plastically. For an increasing bending angle more lattice interactions towards the vertical centre of the domain start to deform plastically.

In Fig. 10 it is visible that the response predicted by the linear QC scheme is slightly stiffer than that of the DLC. The responses of the quadratic and cubic QC frameworks are, somewhat remarkably, slightly more compliant than that predicted by the DLC. Furthermore, the two responses of the quadratic and cubic QC frameworks are virtually the same. The difference between the response of the linear QC framework and that of the DLC is very similar to the difference between the two responses of the quadratic and cubic QC frameworks and that of the DLC.

In Fig. 10 it is furthermore visible that the linear and quadratic QC scheme perform similarly with respect to the prediction when the first lattice interaction reaches a total strain of $15 \%$. The predictions of both QC schemes show a discrepancy of approximately $2 \%$ in applied bending angle when the first interaction reaches $15 \%$, although the linear approach predicts this takes place at a smaller bending angle than the actual one and the quadratic framework predicts it occurs at a larger bending angle. Rather surprisingly the cubic QC framework shows a discrepancy of approximately $4 \%$ in terms of the applied bending angle when the first interaction reaches $15 \%$. In terms of the maximum bending moment however, the quadratic and cubic QC approaches are more accurate than the linear scheme.

In Fig. 11 the relative errors of the total strains of the horizontal interactions are shown for the example of pure bending. The relative errors in Fig. 11 are again shown for the quadratic QC approach with the reference triangulation (third image in Fig. 8) and for increasing fully resolved regions. Similarly to the relative errors predicted for the uniaxial deformation example, the relative errors are smaller for increasing fully resolved domains. 

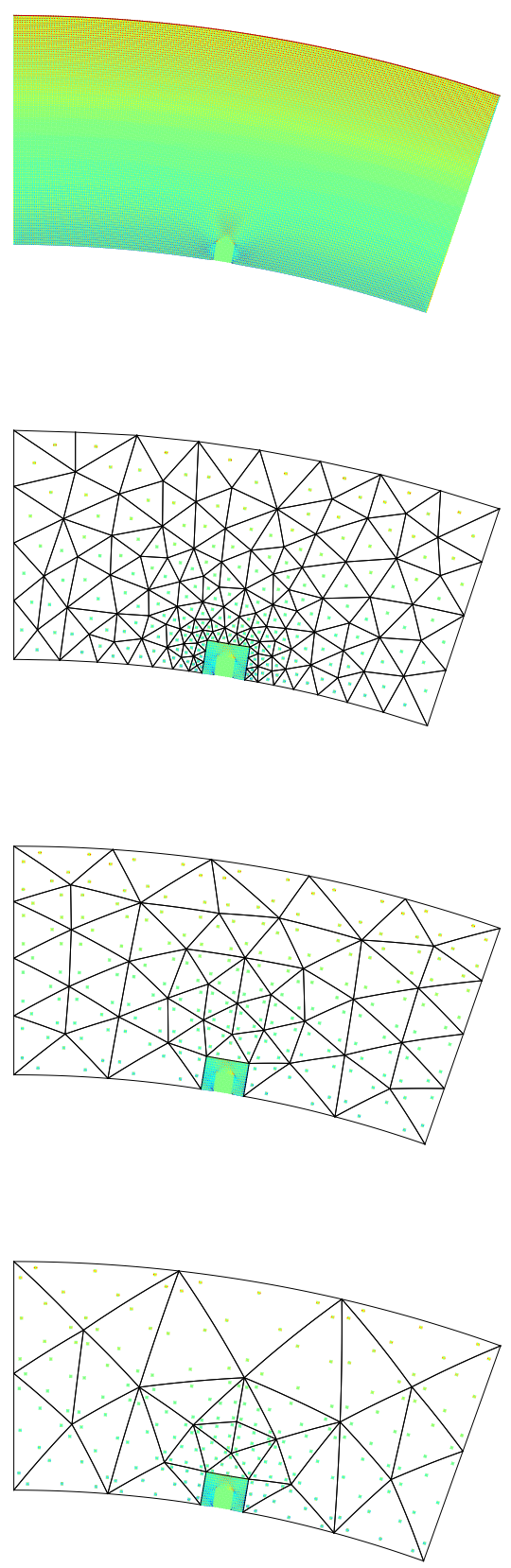

Fig. 8 The total strains of the lattice interactions as predicted by the DLC (top) and of the sampling interactions predicted by the linear QC computation (second), quadratienriged (tine will be protided by the puthlisher cubic QC computation (fourth) for pure bending at the increment at which the total strain in one lattice interaction reaches $15 \%$ for pure bending. Triangles are presented in black. 

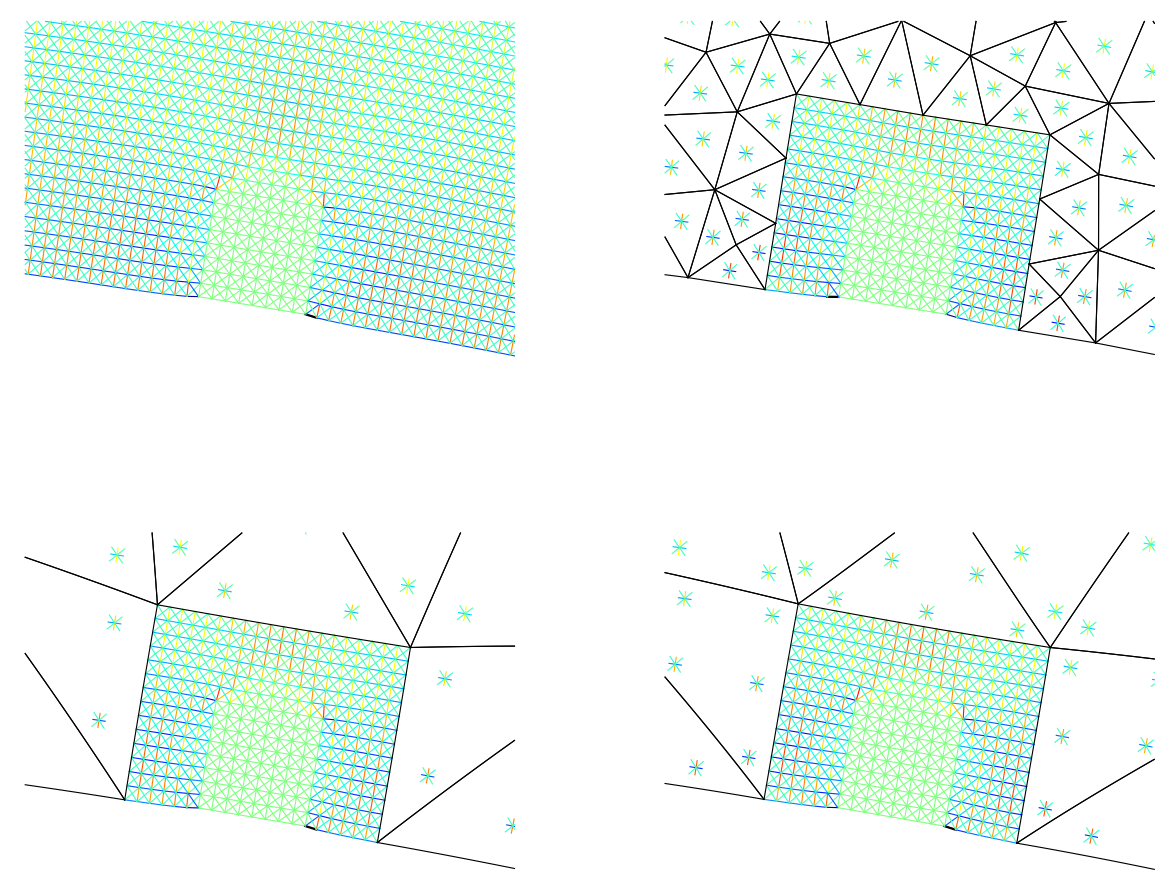

Fig. 9 Enlargements of the stiff region showing the total strains of the lattice interactions as predicted by the DLC (top-left) and of the sampling interactions predicted by the linear QC computation (top-right), quadratic QC computation (bottom-left) and cubic QC computation (bottom-right) for pure bending at the increment at which the total strain in one lattice interaction reaches $15 \%$. The lattice interaction that reaches a total strain of $15 \%$ is shown in black. Triangles are presented in black.

It can be concluded that for the case of pure bending the quadratic and cubic QC schemes are as accurate as the linear QC scheme. All QC schemes predict a discrepancy compared to the DLC result, but this discrepancy is small.

\section{Conclusion}

The quasicontinuum (QC) method is a multiscale approach to increase the efficiency of microstructural lattice models for macroscale computations. The method is concurrent by nature; it fully resolves the lattice model in small regions with high fluctuations - typically the regions in which interesting mechanical phenomena occur (e.g. the onset of fracture) and one 


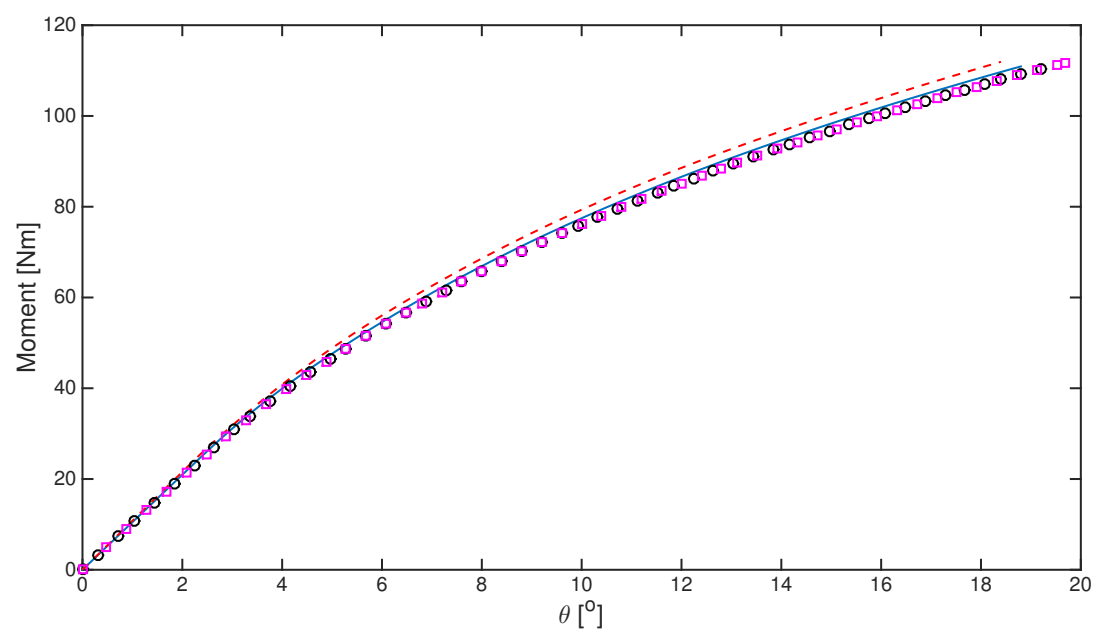

Fig. 10 The moment-angle curves predicted by the DLC (blue, solid), the linear QC computation (red, dashed), the quadratic QC computation (black circles) and the cubic QC computation (magenta squares) for pure bending until one of the lattice interactions reaches a strain of $15 \%$.

desires a high accuracy. Elsewhere, QC methods coarse-grain the lattice model to reduce the computational cost drastically. In the numerical examples in this contribution a speed up of a factor of 40 is achieved. Larger efficiencies can easily be achieved and it depends on the ratio between the size of the fully resolved region and the size of the coarse-grained domain.

The accuracy of three QC schemes with different orders of interpolation is investigated in this contribution (linear, quadratic and cubic). As QC frameworks using higher-order interpolation cannot use conforming triangulations as normally used in the QC method, the lattice models in fully resolved regions are kinematically coupled to coarse domains. Furthermore, sampling interactions are not selected from the physical lattice interactions but they are centred around Gauss quadrature points. The results in this contribution show that local discrepancies in and around the fully resolved regions decrease for increasing sizes of fully resolved domains. This is a beneficial aspect of the presented QC methods, since the user knows that a larger fully resolved domain leads to a more accurate result. This was not be achieved by the central summation rule [19].

The accuracy of the three QC schemes is investigated by comparing their results to those of the direct lattice computations (DLCs). The accuracy is investigated for two numerical examples; one in which macroscopically uniaxial deformation is prescribed and one in which pure bending is macroscopically prescribed. Both multiscale examples are concurrent in nature, since a fully resolved region with an inclusion is incorporated. The QC schemes and the DLCs are compared in such a way that a global quantity has an influence (e.g. total forcedisplacement curve) as well as a microscale quantity (e.g. truncation of the force-displacement curve occurs when one lattice interaction fails). The comparisons of the three QC schemes are made for a similar computational gain compared to the DLC. 

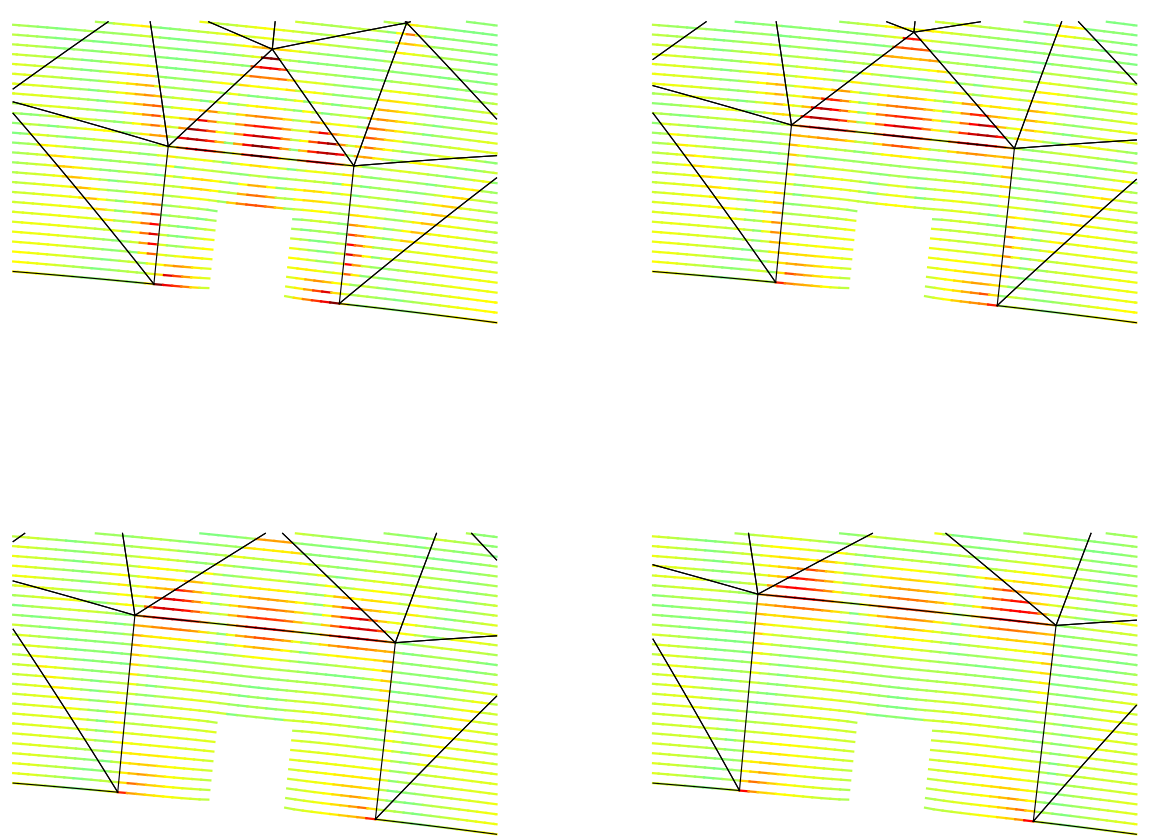

Fig. 11 Enlargements of the stiff region showing the relative errors of the total strains of the horizontal lattice interactions of the quadratic QC framework for the same triangulation but with different sizes of fully resolved domains. The relative errors are determined after increment 150 and hence, at an applied bending angle of $12^{\circ}$. The top-left image shows the relative errors for the reference case (i.e. with a fully resolved region of $20 \times 20$ lattice spacings), the top-right image shows them for a fully resolved region of $24 \times 24$ lattice spacings, the bottom-left image shows them for a fully resolved region of $28 \times 28$ lattice spacings and the bottom-right image shows them for a fully resolved domain of $32 \times 32$ lattice spacings. Triangles are presented in black.

For the uniaxial deformation case, all QC schemes are highly accurate and no distinct difference between the three QC schemes can be observed. The predicted force-displacement curves are virtually identical to that predicted by the DLC. The only difference with the DLC results is that the first lattice interaction is predicted to fail slightly before the first one fails in the DLC.

For the case in which pure bending is prescribed, the QC results are accurate, but not as accurate as for the uniaxial deformation case. The predicted moment-angle curves are for 
instance somewhat less accurate than the predicted force-displacement curves for the uniaxial deformation case. On the other hand, the moments at which the first lattice interaction fails are of a similar accuracy.

The most important conclusion of the current contribution is that higher-order QC approaches are as accurate as linear QC approaches for the same computational gain. It must be mentioned that in this contribution this is only investigated (i) for two numerical examples -uniaxial deformation and pure bending- and (ii) for lattice interactions that have an internally linear interpolation. For lattice interactions that use an internally higher-order interpolation such as beams, the work of [28] has shown that higher-order QC approaches are unavoidable.

\section{Acknowledgements}

Ondřej Rokoš and Jan Zeman would like to acknowledge the financial support from the Czech Science Foundation (project No. 14-00420S).

\section{References}

[1] S. Kato, T. Yoshino, and H. Minami, Eng. Struct. 21, 691-708 (1999).

[2] S. B. Sharma, and M. P. F. Sutcliffe, Composites Part A 35, 637-643 (2004).

[3] D. Durville, Int. J. Mater. Form. 3, 1241-1251 (2010).

[4] L. A. A. Beex, C. W. Verberne, and R. H. J. Peerlings, Composites Part A 48, 82-92 (2013).

[5] A. Kulachenko, and T. Uesaka, Mech. Mater. 51, 1-14 (2012).

[6] J. Persson, and P. Isaksson, Int. J. Numer. Meth. Engng. 93, 1216-1234 (2013).

[7] D. V. Wilbrink, L. A. A. Beex, and R. H. J. Peerlings, Int. J. Solids Struct. 50, 1354-1363 (2013).

[8] E. Bosco, R. H. J. Peerlings, and M. G. D. Geers Int. J. Solids Struct. (2015).

[9] A. Ridruejo, C. Gonzalez, and J. Llorca, J. Mech. Phys. Solids 58, 1628-1645 (2010).

[10] A. Ridruejo, C. Gonzalez, and J. Llorca, Int. J. Multiscale Com. 10, 425-440 (2012).

[11] T. Stylianopoulos, C. A. Bashur, A. S. Goldstein, S. A. Guelcher, and V.H. Barocas, J. Mech. Behav. Biomed. Mater. 1, 326-335 (2008).

[12] G. Argento, M. Simonet, C. W. J. Oomens, and F.P. T. Baaijens, J. Biomech. 45, 2893-2898 (2012).

[13] A. Arslan, R. Ince, and B. L. Karihaloo, J. Eng. Mech. 128, 57-65 (2002).

[14] B. L. Karihaloo, P. F. Shao, and Q.Z. Xiao, Eng. Fract. Mech. 70, 2385-2406 (2003).

[15] R. Vidya Sagar, B. K. Raghu Prasad, and B. L. Karihaloo, Eng. Fract. Mech. 161, 121-129 (2010).

[16] X. Q. Peng, and J. Cao, Composites Part A 36, 859-874 (2005).

[17] E. B. Tadmor, R. Phillips, and M. Ortiz, Langmuir 12, 4529-4534 (1996).

[18] I. G. Kevrekidis, C. W. Gear, and G. Hummer, AIChE Journal 50, 1346-1355 (2004).

[19] L. A. A. Beex, R. H. J. Peerlings, and M. G. D. Geers, J. Mech. Phys. Solids 64, 154-1699 (2014).

[20] L. A. A. Beex, R. H. J. Peerlings, and M. G. D. Geers, Comput. Method. Appl. M. 269, 108-122 (2014).

[21] L. A. A. Beex, R. H. J. Peerlings, K. van Os and M. G. D. Geers, Mech. Mater. 80, 52-66 (2015).

[22] V. B. Shenoy, R. Miller, E. B. Tadmor, R. Phillips, D. Rodney, and M. Ortiz, J. Mech. Phys. Solids 47, 611-642 (1999).

[23] Q. Yang, E. Biyikli, and A. C. To, Comput. Method. Appl. M. 258, 26-38 (2013).

[24] J.S. Amelang, G. N. Venturini, and D. M. Kochmann, Proc. Appl. Math. Mech. 13, 553-556 (2013).

[25] E. Biyikli, Q. Yang, and A. C. To, Comput. Method. Appl. M. 274, $42-55$ (2014).

[26] D. M. Kochmann, and G. N. Venturini, Modelling Simul. Mater. Sci. Eng. 22, 034007 (2014).

[27] X. Wang, X. Guo, and Z. Su, Comput. Method. Appl. M. 268, 284-298 (2014). 
[28] L. A. A. Beex, P. Kerfriden, T. Rabczuk, and S. P. A. Bordas, Comput. Method. Appl. M. 279, 348-378 (2014).

[29] S. Kwon, Y. Lee, J. Y. Park, D. Sohn, J. H. Lim, and S. Im, J. Comput. Phys. 228, 4789-4890 (2009).

[30] B. D. Coleman, W. Noll, Arch. Ration. Mech. Anal. 13, 167-178 (1963).

[31] B. Eidel, and A. Stukowski, J. Mech. Phys. Solids 57, 87-108 (2009).

[32] L. A. A. Beex, R. H. J. Peerlings, and M. G. D. Geers, Int. J. Numer. Meth. Engng. 87, 701-718 (2011).

[33] J. Knap, and M. Ortiz, J. Mech. Phys. Solids 49, 1899-1923 (2001).

[34] C. E. Heaney, L. A. A. Beex, S. P. A. Bordas, and P. Kerfriden, Submitted.

[35] Y. Zhang, and M. Gunzburger, Comput. Method. Appl. M. 199, 648-659 (2010).

[36] M. Gunzburger, and Y. Zhang, Multiscale Model. Simul. 8, 571-590 (2010).

[37] T.J.R. Hughes, The finite element method: linear static and dynamic finite element analysis (Prentice-Hall Inc., Englewood Cliffs, 1987).

[38] N. Triantafyllidis, A. Needleman, and V. Tvergaard, Int. J. Solids Struct. 18, 121-138 (1982).

[39] J. H. Heegaard, and A. Curnier, Int. J. Numer. Meth. Engng. 36, 569-593 (1993). 